\title{
Examining the Role of Self-Regulated Learning on Introductory Programming Performance
}

\author{
Susan Bergin, Ronan Reilly and Desmond Traynor \\ Department of Computer Science, \\ National University of Ireland Maynooth, \\ Maynooth, Co. Kildare. \\ \{sbergin, ronan, dtraynor\}@cs.nuim.ie
}

\begin{abstract}
The purpose of this study was to investigate the relationship between self-regulated learning (SRL) and introductory programming performance. Participants were undergraduate students enrolled in an introductory computer programming module at a third-level (post-high school) institution. The instrument used in this study was designed to assess the motivations and learning strategies (cognitive, metacognitive and resource management strategies) of college students. The data gathered was analyzed to determine if a relationship existed between self-regulation and programming performance and investigate if SRL could be used to predict performance on the module. The study found that students who perform well in programming use more metacognitive and resource management strategies than lower performing students. In addition, students who have high levels of intrinsic motivation and task value perform better in programming and use more metacognitive and resource management strategies than students with low levels of intrinsic motivation and task value. Finally, a regression model based on cognitive, metacognitive and resource management strategies was able to account for $45 \%$ of the variance in programming performance results.
\end{abstract}

\section{Categories and Subject Descriptors}

K.3.2 [Computer and Information Science Education]: Computer science education

\section{General Terms}

Human Factors, Measurement, Performance

\section{Keywords}

CS1, Programming, Predictors, Self-regulated learning

Permission to make digital or hard copies of all or part of this work for personal or classroom use is granted without fee provided that copies are not made or distributed for profit or commercial advantage and that copies bear this notice and the full citation on the first page. To copy otherwise, to republish, to post on servers or to redistribute to lists, requires prior specific permission and/or a fee.

ICER'05, October 1-2, 2005, Seattle, Washington, USA.

Copyright 2005 ACM 1-59593-043-4/05/0010 ...\$5.00.

\section{INTRODUCTION}

Self-regulated learning (SRL) has become an important topic in education and psychology. SRL is defined as the degree to which learners are metacognitively, motivationally and behaviorally active participants in their own academic learning [15]. A considerable number of studies have consistently found a significant positive correlation between academic achievement and self-regulated learning among elementary, high school, and college students [5], [13], [9], [16]. Studies have also found that low self-regulating students are not as academically successful as high self-regulating students. In addition, it has been shown that students who have high task value in a topic (beliefs about the importance of, interest in and utility value of the task) are more likely to use strategies to monitor and regulate their cognition than students with lower task value. Finally, numerous studies have found that an intrinsic goal orientation (undertaking an activity for its own sake, for the enjoyment it provides, the learning it permits, or the feelings of accomplishment it evokes) is strongly positively correlated with the use of cognitive and metacognitive strategies and also with academic performance, [1], [6], [10], [7], [13].

Over the past 30 years a considerable number of studies have taken place to determine factors that influence programming success, for example, [1], [2], [3], [4], [14]. While some studies have had interesting results the area remains largely inconclusive and suggests that perhaps more evidence on potential factors needs to be gathered. It appears that computer science educational researchers have yet to examine, in detail, the role of SRL in learning to program and, more specifically, if SRL is a useful predictor for programming performance. Given this, the purpose of this study is to evaluate the relationship between SRL and programming performance and to determine if SRL can be used as a predictor of introductory programming performance.

\section{SELF-REGULATED LEARNING}

A complete model of self-regulated learning should incorporate cognitive and metacognitive strategies, referred to as a 'skill' component, and motivational components, referred to as 'will' components [9], [16]. Our study is based on a model of self-regulated learning developed by Pintrich and his colleagues [10], [11]. The model includes skill and will components of self-regulated learning. The skill component includes cognitive strategies, metacognitive strategies and resource management strategies. The will component is composed of various motivations, including intrinsic goal ori- 
entation and task value. In this model, the will component is assumed to influence the use of cognitive and metacognitive strategies [8].

Cognitive strategies include rehearsal, elaboration and organizational strategies. Rehearsal strategies include the recitation of information to be learned and mnemonic techniques for memory tasks. These strategies are assumed to help learners to attend to and select important information from lists or texts, but may not reflect a very deep level of processing. Elaboration strategies involve paraphrasing, summarizing, creating analogies and generative note taking. These strategies help learners to integrate and connect new information with prior knowledge. Organizational strategies include clustering, outlining and selecting the main idea from text. These strategies help learners to select appropriate information and to construct connections among the information to be learned [8], [12].

Metacognitive strategies include planning, monitoring and regulating cognition. Planning includes setting goals, skimming a text before reading and analyzing tasks. These activities help to activate relevant aspects of prior knowledge, making the comprehension of the material easier. Monitoring includes tracking one's attention when reading or listening and self-testing using questions. Regulation concerns the continuous modification of one's cognitive activities. For example, a student monitors her attention while reading an article to make certain that she understands its content. When she realizes through her monitoring activities that she has not comprehended a portion of the text, she will go back and reread the difficult part of the article. This rereading of text is a regulation strategy [8], [12]. Finally, resource management strategies refer to strategies students use to manage their time, their effort, their environment and other people, including their interaction with other students and teachers to seek help.

Using this model we will attempt to examine a number of research questions. Of primary importance we would like to investigate if there is a relationship between the use of cognitive, metacognitive and resource management strategies and performance on an introductory programming module. Furthermore, we are interested in examining if there is a relationship between intrinsic motivation or task value and the use of cognitive, metacognitive and resource management strategies or with programming performance. To examine these research questions we have devised a number of hypotheses:

Hypothesis 1: Students who perform well in programming will use more cognitive, metacognitive and resource management strategies than lower performing students.

Hypothesis 2: Students who have high intrinsic motivation will perform better in programming than students with lower intrinsic motivation levels.

Hypothesis 3: Students who have high intrinsic motivation will use more cognitive, metacognitive and resource management strategies than students with lower intrinsic motivation levels.

Hypothesis 4: Students who have higher task value will perform better than students with lower task value.

Hypothesis 5: Students who have higher task value will use more cognitive, metacognitive and resource management strategies than students with lower task value.

Finally, given our interest in developing models to predict programming performance [1], [2], we intend to exam- ine if goal-orientation, task value and use of metacognitive, cognitive and resource management strategies are suitable parameters for predicting performance on an introductory programming course.

\section{METHODS}

The sample consisted of students enrolled in a third level introductory (object-oriented) programming module in the academic year 2004 - 2005. Forty students took the introductory programming module and thirty-five students agreed to participate in our study. The module consists of a one hour lecture, a one hour tutorial and three hours of labs every week. Performance on this module is based on continuous assessment ( $50 \%$ of the overall mark) and a final examination ( $50 \%$ of the overall mark). The measure of performance presented in this paper is the continuous assessment scores for the module. This score is determined by a variety of assessment methods including class tests, lab tests and assignments, all graded by the module lecturer. The fact that the score is determined in this manner assures greater generalization where consistent relations are found. Furthermore, once the end-of-year examination has been completed and graded, a more detailed analysis of performance can be undertaken. The instrument used in this study was the 'Motivated strategies for learning questionnaire' (MSLQ). The MSLQ, was co-designed by Pintrich [12], whose model of SRL we are using in this study, and is grounded in the theory that SRL is a context specific activity which differs from class to class or subject to subject [12]. As this study is attempting to measure SRL in a subject specific situation (programming) the MSLQ was thought to be a suitable tool for measurement.The MSLQ measures 17 different scales: six motivational scales and nine learning strategies. The scales can be used together but given their modular design they can also be administered individually. The MSLQ uses a seven-point scale ranging from 1 (not at all true of me) to 7 (very true of me). In this study we employed scales on the MSLQ that measure:

\section{Value Components}

- Intrinsic goal orientation

- Task value

\section{Cognitive Strategies}

- Rehearsal strategies

- Elaboration strategies

- Organization strategies

\section{Metacognitive Strategies}

- Planning, monitoring and regulating strategies (PMR strategies)

- Critical thinking

\section{Resource Management Strategies}

- Time and study environment strategies (managing and regulating time and study environments)

- Effort regulation strategies (controlling effort and attention in the face of distractions and uninteresting tasks) 
Table 1: Reliability analysis using Cronbach alpha measure

\begin{tabular}{lll}
\hline Scale & $\begin{array}{l}\text { Pintrich } \\
\text { et al [12] }\end{array}$ & $\begin{array}{l}\text { Study } \\
\text { values }\end{array}$ \\
\hline & & \\
$\begin{array}{l}\text { Intrinsic goal orientation } \\
\text { scale }\end{array}$ & .74 & .77 \\
$\begin{array}{l}\text { Task value scale } \\
\text { Rehearsal scale }\end{array}$ & .90 & .87 \\
$\begin{array}{l}\text { Elaboration scale } \\
\text { Organization scale }\end{array}$ & .69 & .68 \\
$\begin{array}{l}\text { Critical thinking scale } \\
\text { Planning, monitoring } \quad \text { and }\end{array}$ & .76 & .60 \\
regulating scale & .64 & .57 \\
$\begin{array}{l}\text { Time and environment scale } \\
\text { Effort regulation scale }\end{array}$ & .76 & .83 \\
$\begin{array}{l}\text { Peer learning scale } \\
\text { Help seeking scale }\end{array}$ & .69 & .77 \\
\hline
\end{tabular}

- Peer learning strategies

- Help seeking strategies.

The study was conducted in the first semester shortly after the students had started programming.

\section{RESULTS}

An a priori analysis was carried out to verify no significant difference existed between the mean overall module scores of the class and the sample. Test assumptions on normality (Kolmogorov-Smirnov and Shapiro-Wilk) were confirmed. Cronbach's alphas for each of the MSLQ sub-scales and the subsequent values calculated in this study are given in Table 1. To test each of the hypotheses Pearson correlation coefficients and one-way ANOVA tests were prepared. Pearson correlation coefficients measure the degree and the direction of the linear relationship between two variables. A one-way ANOVA measures the difference between the means of two or more groups. In the remainder of this section the findings on the relationship between SRL and programming performance is presented, followed by an analysis of the combination of factors that best predicts performance.

\section{Hypothesis 1}

Students who perform well in programming will use more cognitive, metacognitive and resource management strategies than lower performing students. Significant Pearson correlations were found between the use of metacognitive strategies and resource management strategies and are given in Table 2. However, no correlations were found between the use of cognitive strategies and programming performance. Students were categorized according to their level of programming ability (high, medium and low). An ANOVA failed to reveal any statistical differences between the mean scores of students based on their use of cognitive strategies. However, a second ANOVA test did reveal a statistical difference between the mean scores of students based on use of metacognitive strategies $(F(2,31)=6.127, p=0.006)$ and subsequent analysis using Tukey HSD found that students with
Table 2: Significant Pearson correlations ( $r$ ) for MSQL scales with programming performance

\begin{tabular}{lcc}
\hline Measure & N & r \\
\hline & & \\
Critical thinking (CT) & 34 & $.576\left(^{* *}\right)$ \\
$\begin{array}{l}\text { Planning, monitoring and regulat- } \\
\text { ing (PMR) }\end{array}$ & 34 & $\left..462^{* *}\right)$ \\
Total metacognitive strategies & 34 & $.543\left(^{* *}\right)$ \\
Resource strategy: Time & 34 & $.370\left(^{*}\right)$ \\
Resource strategy: Effort & 34 & $.624\left(^{* *}\right)$ \\
Resource strategy: Peer & 34 & $.369\left(^{*}\right)$ \\
Total resource strategy & 34 & $.556\left(^{*}\right)$ \\
** Correlation is significant at the 0.01 level (1-tailed). \\
$*$ Correlation is significant at the 0.05 level (1-tailed).
\end{tabular}

a high level of programming ability used more metacognitive strategies than students with low levels of programming ability. In addition, a third ANOVA test revealed a difference between the mean scores of students based on their use of resource management strategies $(F(2,31)=5.094$, $p=0.012)$ and Tukey HSD indicated that, as with metacognitive strategies, students with high levels of programming ability reported using more resource management strategies than students with low levels of programming ability. Our analysis partly supports hypothesis 1 . It would appear that usage of metacognitive and resource management strategies are important for programming performance while usage of cognitive strategies are not.

\section{Hypothesis 2}

Students who have high intrinsic motivation will perform better in programming than students with lower intrinsic motivation levels. A significant Pearson correlation was found between the intrinsic motivation scale and programming performance $r=0.53, p<0.01$. Students were categorized according to their levels of intrinsic motivation (high, medium and low) and an ANOVA test revealed significant differences between the mean programming scores of the groups $(F(2,31)=4.161, p=0.025)$. Tukey HSD revealed that students with high levels of intrinsic motivation had a statistically higher programming mean score than students with low levels of intrinsic motivation. The evidence gathered supports hypothesis 2 that students with high intrinsic motivation perform better in programming than student with low intrinsic motivation.

\section{Hypothesis 3}

Students who have high intrinsic motivation will use more cognitive, metacognitive and resource management strategies than students with lower intrinsic motivation levels. Significant Pearson correlations were found between responses to the intrinsic goal orientation scale and the use of cognitive, metacognitive and resource management strategies, as shown in Table 3. An ANOVA test failed to reveal a difference in the use of cognitive strategies of students categorized by level of intrinsic motivation (high, medium or low). However it did tend towards a significant difference in cognitive strategy use, $(F(2,31)=3.055, p=0.062)$. Further ANOVA tests did reveal significant differences on the use of 
Table 3: Significant Pearson correlations ( $r$ ) for intrinsic goal orientation with cognitive, metacognitive and resource management strategies

\begin{tabular}{lcc}
\hline Measure & $\mathbf{N}$ & $\mathbf{r}$ \\
\hline & & \\
Cognitive strategies & 34 & $.393\left(^{*}\right)$ \\
Metacognitive strategies & 34 & $.669\left(^{* *}\right)$ \\
$\begin{array}{l}\text { Resource management } \\
\text { strategies }\end{array}$ & & $.42\left(^{* *}\right)$ \\
** Correlation is significant at the 0.01 level (1-tailed). \\
$*$ Correlation is significant at the 0.05 level (1-tailed). \\
\hline
\end{tabular}

metacognitive strategies $(F(2,31)=17.69, p=0.00)$ and resource management strategies $(F(2,31)=5.016, p=0.013)$ of students with different levels of motivation. Post hoc analysis using Tukey HSD found that highly intrinsically motivated students use more metacognitive strategies than students with medium and low levels of intrinsic motivation. In addition, highly intrinsically motivated students use more resource management strategies than students with low levels of intrinsic motivation. The findings partially support hypothesis 3 . Students who have high intrinsic motivation use more metacognitive and resource management strategies than students with lower intrinsic motivation levels but there is no difference in cognitive strategy usage based on intrinsic motivation levels.

\section{Hypothesis 4}

Students who have higher task value will perform better than students with lower task value. A significant Pearson correlation was found between the task value scale and programming performance $r=0.544, p<0.01$. An ANOVA test found a statistical difference between the programming performance of students with different levels of task value $(F(2,31)=6.170, p=0.006)$ and Tukey HSD revealed that students with high levels of task value performed better than students with low task value. The findings support hypothesis 4.

\section{Hypothesis 5}

Students who have higher task value will use more cognitive, metacognitive and resource management strategies than students with lower task value. Significant Pearson correlations were found between responses to the task value scale and the use of cognitive, metacognitive and resource management strategies, as shown in Table 4. An ANOVA test failed to reveal a difference in the use of cognitive strategies of students categorized by level of task value (high, medium or low), however, as with the intrinsic motivation findings, the results did tend towards significant, $(F(2,31)=3.151$, $p=0.057)$. An ANOVA test revealed a significant difference on the use of metacognitive strategies $(F(2,31)=11.87, p=$ $0.00)$ and resource management strategies $(F(2,31)=8.782$, $p=0.01$ ) of students with different levels of task value. Post hoc analysis using Tukey HSD found that students with high levels of task value use more metacognitive strategies than students with medium levels of task value or low levels of task value. Furthermore, students with high task value and students with medium levels of task value use more resource
Table 4: Significant Pearson correlations ( $r$ ) for task value with cognitive, metacognitive and resource management strategies

\begin{tabular}{lcc}
\hline Measure & $\mathbf{N}$ & $\mathbf{r}$ \\
\hline & & \\
Cognitive strategies & 34 & $.347\left(^{*}\right)$ \\
Metacognitive strategies & 34 & $.647\left(^{* *}\right)$ \\
Resource management strategies & 34 & $.636\left(^{* *}\right)$ \\
** Correlation is significant at the 0.01 level (1-tailed). & * Correlation is significant at the 0.05 level (1-tailed). \\
\hline
\end{tabular}

management strategies than students with low task value. The findings partially support hypothesis 5 . Students who have high task value use more metacognitive and resource management strategies than students with lower task value levels but there is no difference in cognitive strategy usage based on task value levels.

\section{Regression Analysis}

To investigate whether the various factors studied were predictive of performance on the module a number of regression models were developed. Each model was motivated by the literature review, the authors' experience working with first year students and the strength of the correlation coefficients generated in this study.

In the first model consideration was given to the use of cognitive strategies and metacognitive strategies. Using a stepwise regression method a significant model emerged with $F(1,32)=13.37, p<0.001$ with an adjusted $\mathrm{R}$ square $=$ $27 \%$. The only significant variable in the model was the metacognitive strategies. Considering, the use of resource management strategies as well, resulted in a slightly more significant model, $F(1,32)=14.35, p<0.001$ with an adjusted $\mathrm{R}$ square $=29 \%$. Given the importance of intrinsic motivation, this regression model was extended to include student's intrinsic goal orientation. This model resulted in an adjusted $\mathrm{R}$ square $=38 \%$ with $F(1,30)=11.066$, $p<0.001$. Task value does not add any further weight to the models. Expanding this model to incorporate the scales within each of the categories (cognitive, metacognitive and resource management strategies) results in a regression model, with an adjusted $\mathrm{R}$ square $=45 \%, F(1,32)=14.281$, $p<0.001$, with two significant variables, effort regulation and critical thinking.

\section{DISCUSSION}

We hypothesized that students who perform well in programming will use more cognitive, metacognitive and resource management strategies than lower performing students. We also expected that students who have high intrinsic motivation will perform better and use more cognitive, metacognitive and resource management strategies than students with lower intrinsic motivation levels. We hypothesized that the same would be true of students who have higher task value for learning programming. The results of our study appear to at least partially support all of our hypotheses. 
The finding that students who use high levels of metacognitive and resource management strategies achieve statistically higher results than students with lower levels of these strategies is consistent with previous research [5], [13], [9], [16]. However, the finding that the use of cognitive strategies does not appear to relate to performance is interesting and warrants further research. Perhaps strategies like rehearsal, elaboration and organization are not as useful in learning introductory object-oriented programming as they are in other other academic domains. A more fine-grained analysis, even to the level of neuroscientific data, for example, using electroencephalogram (EEG), may help to further explain this finding.

The result of this study that students with high levels of task value and intrinsic goal orientation use more selfregulating strategies and are more academically successful (in this instance in introductory programming) is consistent with results from other previous studies, for example [6], [10], [7], [13].

It would appear that specifically designed tools that help students to self-regulate their learning and to encourage student's to develop an intrinsic goal orientation and higher task values might enable them to achieve higher results and to promote their SRL development. However, while this study found a significant relationship between intrinsic motivation, task value and the use of various self-regulated learning strategies, it did not establish a causal explanation for the effects of these different motivational components on the use of learning strategies. It is therefore possible that the successful use of learning strategies caused an increase in programming value and goal orientation and not vice versa. Further research is needed to try to determine the direction of this relationship before any solid recommendations on how to promote the use of student self-regulating strategies or strategies to encourage particular motivational components can be made.

Finally, the predictive ability of SRL for programming performance is a useful contribution for research on factors that influence programming success. Combining SRL with other academic or non academic factors that have already been shown to be predictive, for example the work of, [2], [3], [4], [14], could facilitate the development of a more accurate model for predicting performance on an introductory programming module.

This study has a number of limitations. First of all, as it was a once-off study, repeat studies using similar parameters are required to confirm the findings. To this end, the authors have undertaken to repeat this study at three other institutions. A second limitation of this study is that the measures involved self-report of behaviors. Studies that utilize multiple sources of data and other types of measures, including interviews, observations and think-aloud sessions may help to further explain the nature of the relationship between self-regulated learning and programming performance.

In essence, the most significant contribution of this study is that it establishes that SRL does play a role in learning to program and, as such, it encourages further, more detailed, work in this area.

\section{CONCLUSIONS}

The present study focused on examining the relationship between SRL and performance in an introductory programming module. The results of this study indicate that SRL is important in learning how to program and can be used to partially predict performance on an introductory programming module. The positive findings from this initial investigation warrant further research and provide suggestions for future related studies. For example, a future study could examine if the motivations and learning strategies of students change as they learn to program and, if this is the case, can this change be used to predict programming performance?

\section{ACKNOWLEDGEMENTS}

The authors would like to express their sincere thanks to Margaret Kinsella, Brian Nolan and Larry Mc Nutt for facilitating this study. The authors are also very grateful to the students at the Institute of Technology Blanchardstown who participated in this study.

\section{REFERENCES}

[1] S. Bergin and R. Reilly. The influence of motivation and comfort-level on learning to program. In Proceedings of the 17th Workshop on Psychology of Programming, PPIG'05, 2005.

[2] S. Bergin and R. Reilly. Programming: Factors that influence success. In Proceedings of the 36th SIGCSE technical symposium on computer science education, SIGCSE'05, pages 411-415, 2005.

[3] P. F. Campbell and G. P. McCabe. Predicting the success of freshmen in a computer science major. Commun. ACM, 27(11):1108-1113, 1984.

[4] B. Cantwell-Wilson and S. Shrock. Contributing to success in an introductory computer science course: a study of twelve factors. In Proceedings of the thirty-second SIGCSE technical symposium on Computer Science Education, pages 184-188, 2001.

[5] F. Pajares, S. Brinter, and G. Valiante. Relation between achievement goals and self-beliefs of middle school students in writing and science. Contemporary Educational Psychology, 25(4):406-422, 2000.

[6] H. Patrick, A. Ryan, and P. Pintrich. The differential impact of extrinsic and mastery goal orientations on males' and females' self-regulating learning. Learning and Individual differences, 11(2):153-171, 1999.

[7] P. Pintrich. The dymanic interplay of student motivation and cognition in the college classroom. Advances in motivation and achievement: motivation enhancing environments, 6:117-160, 1989.

[8] P. Pintrich. The role of motivation in promoting and sustaining self -regulated learning. International Journal of Educational Research, 31:459-470, 1999.

[9] P. Pintrich and E. DeGroot. Motivational and self-regulated learning components of classroom academic performance. Journal of Educational Psychology, 82(1):33-40, 1990.

[10] P. Pintrich and T. Garcia. Student goal orientation and self-regulation in the college classroom. Advances in Motivation and Achievement, 7:371-403, 1991.

[11] P. Pintrich and B. Schrauben. 'Students' motivational beliefs and their cognitive engagement in classroom academic tasks' in 'Student perceptions in the classroom'. Hillsdale, NJ:Lawrence Erlbaum, 1992.

[12] P. R. Pintrich, D. Smith, T.Garcia, and W. McKeachie. A manual for the use of the motivated 
strategies for learning questionnaire. technical report 91-b-004. The Regents of the University of Michigan., 1991.

[13] P. Pokay and P. Blumenfeld. Predicting achievement early and late in the semester: the role of motivation and learning strategies. Journal of Educational Psychology, 82(1):41-50, 1990.

[14] N. Rountree, J. Rountree, and A. Robins. Predictors of success and failure in a cs1 course. SIGCSE Bull., 34(4):121-124, 2002.
[15] B. Zimmerman. Becoming a self-regulated learner: which are the key sub-processes? Contemporary Educational Psychology., 11(4):307-313, 1986.

[16] B. Zimmerman and M. Martinez-Pons. Student differences in self-regulated learning: relating grade, sex and giftedness to self-efficacy and strategy use. Journal of Educational Psychology, 82:51-59, 1990. 